\title{
Maize (Zea Mays L.) Growth and Metabolic Dynamics with Plant Growth- Promoting Rhizobacteria under Salt Stress
}

\author{
Abd El-Ghany TM*, Masrahi YS, Mohamed A, Al Abboud, Alawlaqi MM and Nadeem I Elhussieny
}

Biology Department, Faculty of Science, Jazan University, 114, KSA

\begin{abstract}
Maize (Zea mays L.) biomass and its allied attributes were assessed under salinity stress and three plant growthpromoting rhizobacteria (Pseudomonas fluorescens, Pseudomonas putida and Azotobacter vinelandii) treatments. The three PGPRs inocula exhibited a different pattern of shoot growth under both normal and saline stress conditions. Plant biomass, carbohydrates, protein and chlorophyll content were reduced by saline stress, however application of PGPRs treatments improved them either in comparison to control samples or to untreated samples under saline stress. Lipids and antioxidant enzymes (catalase and peroxidase) increased as a response for saline stress as an indication of oxidative stress. Plant growth-promoting rhizobacteria treatment restored them to semi-normal levels. Sodium/ potassium balance was observed to be disturbed by saline stress through higher levels of $\mathrm{Na}^{+}$and lower levels of $\mathrm{K}^{+}$, but treating samples balance was clearly restored close to normal conditions especially in the root system.
\end{abstract}

Keywords: Zea mays L.; Plant growth-promoting rhizobacteria; Salt stress

\section{Introduction}

Biofertilizers diminish the need for expensive chemical fertilizers in crop farming systems because of they are an inexpensive source of nitrogen that increase crop yields. Thus the extensive use of biofertilizers would provide economic benefits to farmers, improve the socio-economic condition of the people and preserve natural resources. Biofertilizers are ecofriendly inputs and are less damaging to the environment when compared to chemical fertilizers $[1,2]$. Beneficial rhizobacteria, often referred to as plant growth-promoting rhizobacteria (PGPR), affect plant growth either directly or indirectly through various mechanisms of action [3-6]. Although, the mechanisms by which PGPRs promote plant growth are not fully understood, some mechanisms include gibberellic acid and/or cytokinins production, nitrogen fixation, and solubilization of mineral phosphate and other nutrients [7].

Maize (Zea mays L.) is the third most important cereal after wheat and rice all over the world [8]. Subramaniyan et al. [9] stated, "the application of biofertilizers improved the total carbohydrate, protein, amino nitrogen and chlorophyll content of of Zea mays". Five growthpromoting strains (Azotobacter sp. Lx191, Pseudomonas sp. Jm92, Bacillus sp. LM4-3, Bacillus sp.LH12-3, and Azospirillum sp. LHS11) were previously isolated from rhizosphere of wheat, maize, oat in arid fields,. These strains were proofed to stimulate these plant growth under controlled conditions via in vitro and pot experiments $[10,11]$.

Soil salinity decreases plant growth, reduces photosynthetic activity and results in nutrient imbalance in plants.It was reported that PGPR significantly increased shoot/root fresh weight, shoot/root dry weight, chlorophyll $\mathrm{a}, \mathrm{b}$ and cartenoid contents of maize under salt stress. PGPR can induce plant tolerance to salinity by producing various hormones and enhancing the availability of nutrients from the soil matrix [12]. Hasnain and Sabri [13] reported that inoculation with Pseudomonas sp. stimulated plant growth by reduction of toxic ion uptake and production of stress-specific proteins in plant. PGPR strains can also produce exopolysaccharides (EPSs) to bind cations including sodium, thus help alleviating salt stress in plants grown under saline environment [14]. The rhizosphere is the soil portion found around the root and under the influence of the root. It is the site with complex interaction between the root and associated microorganisms [15]. The rhizosphere harbors a multitude of microorganisms that are affected by both abiotic and biotic stresses. Among these are the dominant rhizobacteria that prefer living in close vicinity to the root or on its surface and play a crucial role in soil health and plant growth $[16,17]$. It has been noted by many workers that Pseudomonas, Bacillus, Arthrobacter, Azospirillum, Klebsiella, and Enterobacter, isolated from the rhizosphere of various crops, showed synergistic effects on plant growth [18]. Weller [19] reported that PGPR belong to several genera, e.g. Agrobacterium, Alcaligenes, Arthrobacter, Actinoplanes, Azotobacter, Bacillus, Pseudomonas sp., Rhizobium, Bradyrhizobium, Erwinia, Enterobacter, Amorpho sporangium, Cellulomonas, Flavobacterium, Streptomyces and Xanthomonas. These groups of bacteria are important as they are involved in various soil biochemical processes such as fixation of atmospheric nitrogen, solubilization of minerals such as phosphorus, production of siderophores that solubilize and sequester iron and/or production of plant growth regulators [20]. Nitrogen-fixing bacteria are widely distributed in nature where they reduce atmospheric nitrogen in soil or in association with plant [21]. They have been found in a wide variety of terrestrial and aquatic habitats in both temperate and tropical regions of the world [22]. Biofertilizer contains living microorganisms and promotes growth by increasing the availability of primary nutrients (nitrogen and phosphorus) to the host plant [2326]. Among the free-living nitrogen-fixing bacteria those belonging to genus Azotobacter play a remarkable role, being broadly dispersed in

*Corresponding author: Abd El-Ghany TM, Biology Department, faculty of Science, Jazan University, KSA, E-mail: tabdelghany@yahoo.com

Received September 16, 2015; Accepted September 28, 2015; Published September 30, 2015

Citation: Abd El-Ghany TM, Masrahi YS, Mohamed A, Abboud A, Alawlaqi MM et al. (2015) Maize (Zea Mays L.) Growth and Metabolic Dynamics with Plant Growth-Promoting Rhizobacteria under Salt Stresses. J Plant Pathol Microb 6: 305. doi:10.4172/2157-7471.1000305

Copyright: (C) 2015 Abd El-Ghany TM, et al. This is an open-access article distributed under the terms of the Creative Commons Attribution License, which permits unrestricted use, distribution, and reproduction in any medium, provided the original author and source are credited. 
Citation: Abd El-Ghany TM, Masrahi YS, Mohamed A, Abboud A, Alawlaqi MM, et al. (2015) Maize (Zea Mays L.) Growth and Metabolic Dynamics with Plant Growth-Promoting Rhizobacteria under Salt Stresses. J Plant Pathol Microb 6: 305. doi:10.4172/2157-7471.1000305

different environments, such as soil, water and sediments [27]. Several authors have shown the beneficial effects of Azotobacter chroococcum on vegetative growth and yields of maize [27-29], as well as the positive effect of inoculation with this bacterium on wheat [30]. Bacillus group was the most dominant strain found in the three types of biofertilizer products. The other bacteria were Azospirillum, Corynebacterium, Pseudomonas and Proteus mirabilis. These bacteria have the potential to fix atmospheric nitrogen, able to produce IAA with the supplemented tryptophan, and showed some phosphorus solubilizing activity [15,31]. The aim of this work was to assess the effect of PGPR on the growth of Z. mays. Also it was carried out to elucidate the role of PGPR on growth and ion uptake of maize under salt stress condition.

\section{Materials and Methods}

\section{Description of the study area}

The present study focused on the area in Jazan. The study area is situated in Jazan, Kingdom of Saudi Arabia (Lat. 16 $53^{\prime} 21^{\prime \prime}$ N , Long. $42^{\circ} 33^{\prime} 03^{\prime \prime} \mathrm{E}$ and $19 \mathrm{~m}$ Elevation above sea level) with the significant features of evergreen forests and also it was a less explored ecosystem for the investigation of biofertilizers population.

\section{Bacterial strains used}

Plant growth-promoting rhizobacteria were isolated from maize rhizosphere growing in Jazan area. Selection of isolates was performed on the basis of the PGPR traits. Selected isolates identified according standard microbiological methods as described in Bergys Manual of Systematic Microbiology [32]. The physiological and biochemical characters, included: starch hydrolysis, gelatin liquefaction, indole production, nitrate reduction, urease activity, citrate utilization, production of oxidase, catalase, methyl red, voges proskauer, tryptophane deaminase, gelatinase, lysine decarboxylase, arginine dihydrolase, B-galactosidase and fermentation oxidation of the following carbon sources (D-glucose, D-mannitol, inositol, D-sorbitol, rhamnose, D-sucrose, D-melibiose maltose, fructose, inulin and Larabinose) were used for identification of bacterial isolates. Bacterial isolates were grown on yeast manitol agar (YMA) supplemented with different concentrations of $\mathrm{NaCl}$ for salt tolerance test. Identification of highly $\mathrm{NaCl}$ tolerance was done using biochemical analysis. Three isolates including Pseudomonas fluorescens, Azotobacter vinelandii and Pseudomonas putida were used in this study.

\section{Inoculums preparation and Zea mays L. growth experimental design}

Fresh cultures of selected isolates were inoculated in pikovisky media and shaken at $37^{\circ} \mathrm{C}$ for two days in an orbital shaker at $100 \mathrm{rpm}$. After two days bacterial cultures were centrifuged at $3000 \mathrm{rpm}$ for 15 mins. Maize (Z. mays L.) grains were surface sterilised with $0.5 \%$ $(\mathrm{v} / \mathrm{v}) \mathrm{NaOCl}$ for $10 \mathrm{~min}$ and were subsequently washed with sterilized deionised water. The sterilized grains were soaked in distilled water in case of un-inoculated control. The rest of sterilized seeds were soaked in broth cultures of isolates form $4-5 \mathrm{hr}$ prior to sowing at two different concentrations $10^{4}$ (low concentration) and $10^{8}$ (high concentration) bacterial cells. Grains were germinated in plastic pots $(15 \mathrm{~cm}$ diameter) with $2 \mathrm{~kg}$ sterilized soil. After sowing, seedlings were reduced to three per pot. The pots were treated as the following: 1) Untreated control, 2) $10^{4} / \mathrm{ml}$ bacterial cell suspension, 3$) 10^{8} / \mathrm{ml}$ bacterial cell suspension, 4) $35 \mathrm{mM} \mathrm{Na} \mathrm{Cl}$ solution, 5) $70 \mathrm{mM} \mathrm{Na} \mathrm{Cl}$ solution, 6) $35 \mathrm{mM} \mathrm{Na} \mathrm{Cl}$ $+10^{4} / \mathrm{ml}$ bacterial cell mixture, 7) $35 \mathrm{mM} \mathrm{Na} \mathrm{Cl}+10^{8} / \mathrm{ml}$ bacterial cell mixture, 8) $70 \mathrm{mM} \mathrm{Na} \mathrm{Cl}+10^{4} / \mathrm{ml}$ bacterial cell mixture and 9) $70 \mathrm{mM}$

\section{$\mathrm{Na} \mathrm{Cl}+10^{8} / \mathrm{ml}$ bacterial cell}

Growth parameters including dry weight, height, leaves length and width of Maize plants were recorded.

\section{$\mathrm{Na}^{+}$and $\mathrm{K}^{+}$content analysis}

Oven - dried samples of Zea mays roots and shoots were powdered for estimation of $\mathrm{Na}^{+}$and $\mathrm{K}^{+}$by flame photometric method [33].

\section{Quantitative determination of chlorophyll}

Chlorophylls content was determined with using the following equations:

$$
\begin{aligned}
& \text { Chlorophyll a (mg)/tissue (g) }=11.63 \text { (A 665) -2.39 (A 649) } \\
& \text { Chlorophyll b (mg)/tissue (g) }=2.11 \text { (A 649) -5.18 (A 665) }
\end{aligned}
$$

Where A denotes the reading of the optical density.

Antioxidant enzymes catalase and peroxidase of healthy and infected plant were determined according to Kar and Mishra [34].

\section{Estimation of Total protein content}

Total protein was estimated calorimetrically [35] by recording absorbance at $595 \mathrm{~nm}$. Bovine serum albumin was used as standard. Protein content in plant samples was recorded as $\mathrm{mg}$ of protein per $\mathrm{g}$ of sample.

\section{Estimation of Total carbohydrate content}

Plant extract was taken in $25 \mathrm{ml}$ test tubes and $6 \mathrm{ml}$ anthrone reagent ( $150 \mathrm{mg}$ of anthrone in $72 \% \mathrm{H}_{2} \mathrm{SO}_{4}$ ) was added, and then heated in boiling water bath for $10 \mathrm{~min}$. The test tubes were ice cooled for $10 \mathrm{~min}$ and incubated for $20 \mathrm{~min}$ at $25^{\circ} \mathrm{C}$. Optical density (OD) was read at 625 $\mathrm{nm}$ on a spectrophotometer. The carbohydrate content was calculated from the standard curve using glucose with the same method which mentioned above [36].

\section{Estimation of total lipid content}

Total lipid was estimated using Vanillin reagent (6.1 g of vanillin was dissolved in water and diluted to 1 liter). The OD was read on a spectrophotometer at $540 \mathrm{~nm}$. The lipid content was calculated from the standard curve using standard solution of cholesterol with the same method which mentioned above.

\section{Results \\ Plant biomass growth}

Maize biomass in terms of plant height, stem diameter, leaf surface area, and plant dry weight was investigated in relation to saline stress $(0,35$, and $70 \mathrm{mM})$ and PGPRs (Azotobacter vinelandii, Pseudomonas fluorescens and Pseudomonas putida) treatments. Plant height (Table 1) was reduced from $103.33 \mathrm{~cm}$ to $91 \mathrm{~cm}$ by saline stress in untreated samples. PGPRs treatments improved plant height in both stressed and normal conditions. A. vinelandii at both their concentrations significantly increased plant height from 103.33 to $144.33 \mathrm{~cm}$ under normal conditions. Under saline stress A. vinelandii increased plant height at $35 \mathrm{mM}$ by $33.77 \%$ and $13.55 \%$ at $70 \mathrm{mM}$ saline stress. $P$. fluorescens was inferior to $A$. vinelandii as it was able to increase plant height in untreated samples to $131 \mathrm{~cm}$ in normal conditions while it was $29.17 \%$ more than untreated sample at $35 \mathrm{mM}$ saline stress and no significant increase at $70 \mathrm{mM}$. P. putida did not improved plant height significantly both in normal and stressed conditions. 
Citation: Abd El-Ghany TM, Masrahi YS, Mohamed A, Abboud A, Alawlaqi MM, et al. (2015) Maize (Zea Mays L.) Growth and Metabolic Dynamics with Plant Growth-Promoting Rhizobacteria under Salt Stresses. J Plant Pathol Microb 6: 305. doi:10.4172/2157-7471.1000305

Page 3 of 9

\begin{tabular}{|c|c|c|c|c|}
\hline \multirow{2}{*}{\multicolumn{2}{|c|}{ PGPR(Cell ml-1) }} & \multicolumn{3}{|c|}{ Saline stress (mM) } \\
\hline & & 0 & 35 & 70 \\
\hline \multicolumn{2}{|l|}{ Control } & $103.33 \pm 1.45 \mathrm{cdef}$ & $101.67 \pm 9.28 \mathrm{def}$ & $91.00 \pm 3.79 f$ \\
\hline \multirow{2}{*}{ A. vinelandii } & $10^{4}$ & $132.33 \pm 7.84 a b$ & $114.33 \pm 5.33 \mathrm{bcde}$ & $102.67 \pm 7.06 \mathrm{cdef}$ \\
\hline & $10^{8}$ & $144.33 \pm 4.37 a$ & $136.00 \pm 6.51 \mathrm{ab}$ & $103.33 \pm 11.67 \mathrm{cdef}$ \\
\hline \multirow{2}{*}{$P$. fluorescens } & $10^{4}$ & $125.00 \pm 2.89 a b c$ & $100.67 \pm 8.35$ def & $89.33 \pm 7.31 f$ \\
\hline & $10^{8}$ & $131.00 \pm 4.58 a b$ & $131.33 \pm 10.27 a b$ & $92.33 \pm 8.88 \mathrm{ef}$ \\
\hline \multirow{2}{*}{ P. putida } & $10^{4}$ & $119.33 \pm 1.86 \mathrm{bcd}$ & $105.67 \pm 7.45 \mathrm{cdef}$ & $85.33 \pm 2.03 f$ \\
\hline & $10^{8}$ & $119.33 \pm 3.84 \mathrm{bcd}$ & $115.67 \pm 10.84 \mathrm{bcd}$ & $98.67 \pm 2.96 \mathrm{def}$ \\
\hline
\end{tabular}

Means followed by the same letter are not significantly different.

Table 1: Mean comparison and standard error of maize plant height $(\mathrm{cm})$ under saline stress and PGPRs treatment.

\begin{tabular}{|c|c|c|c|c|}
\hline \multirow{2}{*}{\multicolumn{2}{|c|}{ PGPR(Cell ml-1) }} & \multicolumn{3}{|c|}{ Saline stress (mM) } \\
\hline & & 0 & 35 & 70 \\
\hline \multicolumn{2}{|l|}{ Control } & $2.93 \pm 0.12 \mathrm{efg}$ & $2.80 \pm 0.06 \mathrm{fg}$ & $2.17 \pm 0.19 g$ \\
\hline \multirow{2}{*}{ A. vinelandii } & $10^{4}$ & $4.07 \pm 0.22 \mathrm{abcde}$ & $3.43 \pm 0.23 \mathrm{cdef}$ & $2.63 \pm 0.19 f g$ \\
\hline & $10^{8}$ & $4.67 \pm 0.38 a b$ & $3.57 \pm 0.23$ bcdef & $3.23 \pm 0.46 \mathrm{defg}$ \\
\hline \multirow{2}{*}{ P. fluorescens } & $10^{4}$ & $4.27 \pm 0.15 a b c d$ & $3.60 \pm 0.15 b c d e f$ & $3.07 \pm 0.35 \mathrm{defg}$ \\
\hline & $10^{8}$ & $4.80 \pm 0.06 a$ & $4.03 \pm 0.09 a b c d e$ & $3.43 \pm 0.09 \mathrm{cdef}$ \\
\hline \multirow{2}{*}{ P. putida } & $10^{4}$ & $4.60 \pm 0.21 \mathrm{abc}$ & $3.80 \pm 0.44$ abcdef & $2.77 \pm 0.19 f g$ \\
\hline & $10^{8}$ & $4.87 \pm 0.09 a$ & $3.17 \pm 0.67 \mathrm{defg}$ & $3.37 \pm 1.07 \mathrm{def}$ \\
\hline
\end{tabular}

Means followed by the same letter are not significantly different.

Table 2: Mean comparison and standard error of maize stem diameter $(\mathrm{cm})$ under saline stress and PGPRs treatment.

\begin{tabular}{|c|c|c|c|c|}
\hline \multirow{2}{*}{\multicolumn{2}{|c|}{ PGPR(Cell ml-1) }} & \multicolumn{3}{|c|}{ Saline stress (mM) } \\
\hline & & 0 & 35 & 70 \\
\hline \multicolumn{2}{|l|}{ Control } & $231.25 \pm 16.16$ bcdef & $77.00 \pm 3.82 \mathrm{j}$ & $81.85 \pm 17.82 j$ \\
\hline \multirow{2}{*}{ A. vinelandii } & $10^{4}$ & $258.68 \pm 14.81 \mathrm{abcde}$ & $157.70 \pm 22.05 f g h i j$ & $128.78 \pm 24.20 h i j$ \\
\hline & $10^{8}$ & $293.08 \pm 25.93 a b c$ & $179.88 \pm 50.12$ efghi & $134.30 \pm 26.04 \mathrm{hij}$ \\
\hline \multirow{2}{*}{ P. fluorescens } & $10^{4}$ & $243.88 \pm 2.99$ abcde & $190.30 \pm 37.53$ defghi & $125.05 \pm 18.71 \mathrm{ij}$ \\
\hline & $10^{8}$ & $312.70 \pm 1.76 a$ & $268.75 \pm 33.11 \mathrm{abcd}$ & $141.60 \pm 17.33 \mathrm{ghij}$ \\
\hline \multirow{2}{*}{ P. putida } & $10^{4}$ & $209.13 \pm 35.58$ defgh & $157.73 \pm 21.21$ fghij & $126.83 \pm 22.48 \mathrm{ij}$ \\
\hline & $10^{8}$ & $298.35 \pm 11.89 a b$ & $217.35 \pm 27.07 \mathrm{cdefg}$ & $152.38 \pm 18.59 f g h i j$ \\
\hline
\end{tabular}

Means followed by the same letter are not significantly different.

Table 3: Mean comparison and standard error of maize leaf surface area $\left(\mathrm{cm}^{2}\right)$ under saline stress and PGPRs treatment.

Stem diameter was not significantly affected by saline stress (Table 2), however it was clearly influenced by PGPRs treatment. The three tested PGPRs (at $10^{4}$ and $10^{8} \mathrm{cell} \mathrm{ml}^{-1}$ ) improve stem diameters by 38 $66 \%$ under no saline stress. P. fluorescens was the best PGPR treatment that enhanced stem diameter from 2.8 to $4.03 \mathrm{~cm}$ at $35 \mathrm{mM}$ saline stress and $10^{8} \mathrm{cell} \mathrm{ml}^{-1}$; while stem diameter was $3.43 \mathrm{~cm}$ at $70 \mathrm{mM}$ saline and $10^{8}$ cell ml-1 against $2.17 \mathrm{~cm}$ at $70 \mathrm{mM}$ saline untreated samples. A. vinelandii treatment improve plant stems diameter by $27.5 \%$ and $48.85 \%$ at 35 and $70 \mathrm{mM}$ saline stress respectively. P. putida both concentrations improve plant stems diameter to 3.8 at low saline stress and 3.37 at the higher one.

Leaf surface area of maize plant was significantly reduced by $66.7 \%$ due to saline stress (Table 3). All tested PGPRs treatment increased plant leaf surface area to $298.35 \mathrm{~cm}^{2}$ under no saline stress. A. vinelandii and $P$. putida treatment limited leaf surface area reduction by saline stress from $66.7 \%$ to range between 31.8 and $6 \%$ at $35 \mathrm{mM}$ stress and between 44.3 and $34.1 \%$ at $70 \mathrm{mM}$. P. fluorescens treatment was superior it was capable of increasing leaf surface area to $268.75 \mathrm{~cm}^{2}$ that is $16 \%$ more than control leaf surface area

Plant dry weight (Table 4) was reduced from $6.4 \mathrm{~g}$ to $3.4 \mathrm{~g}$ by saline stress in untreated samples. PGPRs treatment improve plant dry weight in both stressed and normal conditions. A. vinelandii both concentrations significantly increased plant dry weight from $6.4 \mathrm{~g}$ to $11.2 \mathrm{~g}$ under normal conditions. Under saline stress $A$. vinelandii increased plant dry weight at $35 \mathrm{mM}$ to 12.8 g. P. fluorescens was able to increase plant dry weight under no saline stress to $13.1 \mathrm{~g}$ while it was $11.9 \mathrm{~g}$ at $35 \mathrm{mM}$ saline stress and $9.9 \mathrm{~g}$ at $70 \mathrm{mM}$. P. putida improved plant height significantly both in normal and stressed conditions.

\section{Biochemical contents}

Maize plant carbohydrate content (Table 5) was investigated during the current study. However, the differences in carbohydrates content among treatments was limited; it showed high degree of significance. Carbohydrate content was $79.2 \mathrm{mg} / \mathrm{g}$ in the control plant. PGPRs treatments at no saline stress increased carbohydrates content of the plant up to $82.2 \mathrm{mg} / \mathrm{g}$. Although saline stress reduced plant carbohydrate content in all treated and untreated samples as compared to control sample; it is obvious that except of $P$. putida, the other treatments improve plant carbohydrate content as compared to untreated samples (Table 6).

Maize proteins were $26.17 \mathrm{mg} / \mathrm{g}$ in control sample. PGPRs treatments significantly improve plant protein content up to 33.17 $\mathrm{mg} / \mathrm{g}$ under no saline stress. Saline stress reduced maize protein content to 24.17 and $20.17 \mathrm{mg} / \mathrm{g}$ at 35 and $70 \mathrm{mM}$ saline stress, respectively. $A$. vinelandii and $P$. fluorescens treatment improve palnt protein content by $7.5 \%$ as compared to untreated sample at $35 \mathrm{mM}$. PGPRs treatment showed no considerable differences in the plant protein content when compared to untreated samples at $70 \mathrm{mM}$. Unlike other investigated 
Citation: Abd El-Ghany TM, Masrahi YS, Mohamed A, Abboud A, Alawlaqi MM, et al. (2015) Maize (Zea Mays L.) Growth and Metabolic Dynamics with Plant Growth-Promoting Rhizobacteria under Salt Stresses. J Plant Pathol Microb 6: 305. doi:10.4172/2157-7471.1000305

Page 4 of 9

\begin{tabular}{|c|c|c|c|c|}
\hline \multirow{2}{*}{\multicolumn{2}{|c|}{ PGPR(Cell ml-1) }} & \multicolumn{3}{|c|}{ Saline stress (mM) } \\
\hline & & 0 & 35 & 70 \\
\hline \multicolumn{2}{|l|}{ Control } & $6.40 \pm 0.29 f$ & $3.40 \pm 0.23 \mathrm{~h}$ & $4.00 \pm 0.29 h$ \\
\hline \multirow{2}{*}{ A. vinelandii } & $10^{4}$ & $10.00 \pm 0.23 d$ & $11.10 \pm 0.29 c$ & $5.40 \pm 0.29 g$ \\
\hline & $10^{8}$ & $11.20 \pm 0.29 c$ & $12.80 \pm 0.29 b$ & $6.80 \pm 0.35 \mathrm{ef}$ \\
\hline \multirow{2}{*}{ P. fluorescens } & $10^{4}$ & $9.30 \pm 0.17 d$ & $11.93 \pm 0.20 c$ & $5.07 \pm 0.26 \mathrm{~g}$ \\
\hline & $10^{8}$ & $13.10 \pm 0.58 b$ & $11.30 \pm 0.23 c$ & $9.90 \pm 0.06 d$ \\
\hline \multirow{2}{*}{ P. putida } & $10^{4}$ & $7.30 \pm 0.17$ ef & $14.00 \pm 0.29 a$ & $6.20 \pm 0.12 f$ \\
\hline & $10^{8}$ & $13.00 \pm 0.29 b$ & $14.20 \pm 0.23 a$ & $6.80 \pm 0.23 \mathrm{ef}$ \\
\hline
\end{tabular}

Means followed by the same letter are not significantly different.

Table 4: Mean comparison and standard error of maize dry weight $(\mathrm{g})$ under saline stress and PGPRs treatment.

\begin{tabular}{|c|c|c|c|c|}
\hline \multirow{2}{*}{\multicolumn{2}{|c|}{ PGPR(Cell ml-1) }} & \multicolumn{3}{|c|}{ Saline stress (mM) } \\
\hline & & 0 & 35 & 70 \\
\hline \multicolumn{2}{|l|}{ Control } & $79.20 \pm 0.13 f$ & $69.20 \pm 0.13 j$ & $66.20 \pm 0.13 m$ \\
\hline \multirow{2}{*}{ A. vinelandii } & $10^{4}$ & $81.00 \pm 0.13 c$ & $69.95 \pm 0.13 i$ & $67.18 \pm 0.13 \mid$ \\
\hline & $10^{8}$ & $81.69 \pm 0.13 b$ & $70.98 \pm 0.13 h$ & $67.89 \pm 0.13 k$ \\
\hline \multirow{2}{*}{ P. fluorescens } & $10^{4}$ & $80.20 \pm 0.13 e$ & $70.20 \pm 0.13 i$ & $69.89 \pm 0.13 i$ \\
\hline & $10^{8}$ & $82.20 \pm 0.13 a$ & $71.98 \pm 0.13 g$ & $69.94 \pm 0.13 i$ \\
\hline \multirow{2}{*}{ P. putida } & $10^{4}$ & $80.00 \pm 0.13 e$ & $67.95 \pm 0.13 k$ & $65.28 \pm 0.12 n$ \\
\hline & $10^{8}$ & $80.62 \pm 0.12 d$ & $67.58 \pm 0.13 k$ & $62.80 \pm 0.130$ \\
\hline
\end{tabular}

Means followed by the same letter are not significantly different.

Table 5: Mean comparison and standard error of maize carbohydrates $(\mathrm{mg} / \mathrm{g})$ under saline stress and PGPRs treatment.

\begin{tabular}{|c|c|c|c|c|}
\hline \multirow{2}{*}{\multicolumn{2}{|c|}{ PGPR(Cell ml-1) }} & \multicolumn{3}{|c|}{ Saline stress (mM) } \\
\hline & & 0 & 35 & 70 \\
\hline \multicolumn{2}{|l|}{ Control } & $26.17 \pm 0.02 e$ & $24.17 \pm 0.01 \mathrm{~h}$ & $20.17 \pm 0.181$ \\
\hline \multirow{2}{*}{ A. vinelandii } & $10^{4}$ & $28.18 \pm 0.01 d$ & $25.18 \pm 0.06 \mathrm{~g}$ & $20.12 \pm 0.181$ \\
\hline & $10^{8}$ & $32.13 \pm 0.06 b$ & $26.00 \pm 0.02 e$ & $20.93 \pm 0.01 \mathrm{k}$ \\
\hline \multirow{2}{*}{ P. fluorescens } & $10^{4}$ & $30.17 \pm 0.01 c$ & $25.17 \pm 0.06 \mathrm{~g}$ & $21.10 \pm 0.18 \mathrm{k}$ \\
\hline & $10^{8}$ & $33.17 \pm 0.06 a$ & $25.69 \pm 0.02 f$ & $21.99 \pm 0.01 j$ \\
\hline \multirow{2}{*}{ P. putida } & $10^{4}$ & $26.19 \pm 0.01 \mathrm{e}$ & $22.12 \pm 0.06 j$ & $18.94 \pm 0.01 \mathrm{~m}$ \\
\hline & $10^{8}$ & $28.11 \pm 0.07 d$ & $23.33 \pm 0.32 i$ & $20.12 \pm 0.12 \mid$ \\
\hline
\end{tabular}

Means followed by the same letter are not significantly different.

Table 6: Mean comparison and standard error of maize protein $(\mathrm{mg} / \mathrm{g})$ under saline stress and PGPRs treatment.

\begin{tabular}{|c|c|c|c|c|}
\hline \multirow{2}{*}{\multicolumn{2}{|c|}{ PGPR(Cell ml-1) }} & \multicolumn{3}{|c|}{ Saline stress (mM) } \\
\hline & & 0 & 35 & 70 \\
\hline \multicolumn{2}{|l|}{ Control } & $18.65 \pm 0.13 h$ & $19.14 \pm 0.14 \mathrm{~g}$ & $19.65 \pm 0.13 f$ \\
\hline \multirow{2}{*}{ A. vinelandii } & $10^{4}$ & $18.02 \pm 0.06 i$ & $20.41 \pm 0.06 \mathrm{de}$ & $23.05 \pm 0.02 b$ \\
\hline & $10^{8}$ & $20.56 \pm 0.12 d$ & $21.15 \pm 0.01 c$ & $23.95 \pm 0.06 a$ \\
\hline \multirow{2}{*}{ P. fluorescens } & $10^{4}$ & $19.65 \pm 0.12 f$ & $18.65 \pm 0.05 h$ & $18.15 \pm 0.05 i$ \\
\hline & $10^{8}$ & $19.85 \pm 0.14 f$ & $18.45 \pm 0.12 \mathrm{~h}$ & $19.65 \pm 0.12 f$ \\
\hline \multirow{2}{*}{ P. putida } & $10^{4}$ & $17.00 \pm 0.06 j$ & $19.21 \pm 0.07 \mathrm{~g}$ & $21.02 \pm 0.07 c$ \\
\hline & $10^{8}$ & $18.54 \pm 0.12 h$ & $20.15 \pm 0.02 e$ & $20.90 \pm 0.12 c$ \\
\hline
\end{tabular}

Means followed by the same letter are not significantly different.

Table 7: Mean comparison and standard error of maize lipid content $(\mathrm{mg} / \mathrm{g})$ under saline stress and PGPRs treatment.

primary metabolites, lipid content was significantly proportion to saline stress. Lipid content (Table 7) of maize was also improve by PGPRs treatments. A. vinelandii treatment raised lipid content in maiz plant from 18.65 to $20.56 \mathrm{mg} / \mathrm{g}$ at no saline stress, and to $23.95 \mathrm{mg} / \mathrm{g}$ at $70 \mathrm{mM}$ saline stress. P. fluorescens treatment did not increase maize lipid content significantly as compared to untreated samples at no saline stress. P. putida improved lipid content in maize plant at 35 and $70 \mathrm{mM}$ saline stress.

Chlorophyll content of maize plant was investigated in the current study. Under no saline stress A. vinelandii and P. Fluorescens significantly improve chlorophyll a content (Table 8) to 6.99 and 7.99 $\mathrm{mg} / \mathrm{g}$, respectively. Saline stress reduced plant chlorophyll a content in both PGPRs treated and untreated samples. A. vinelandii and $P$. fluorescens treatments significantly limited chlorophyll a content reduction from $46.86 \%$ to 31.59 and $27.99 \%$ at $35 \mathrm{mM}$ saline stress, respectively. Chlorophyll b content (Table 9) was reduced due to saline stress from 3.21 to $2.1 \mathrm{mg} / \mathrm{g}$. PGPRs treatments either did not influence or reduced chlorophyll b content under no saline stress, $35 \mathrm{mM}$ and 70 $\mathrm{mM}$ saline stress.

Antioxidant enzymes as indicators for oxidative stress exerted on the plant were investigated. Saline stress increased catalase concentration (Figure 1) in untreated and PGPRs treated samples. PGPRs treatments reduced the amount of catalase in plants under saline stress when compared to untreated stressed plants. Salinity stress 
Citation: Abd El-Ghany TM, Masrahi YS, Mohamed A, Abboud A, Alawlaqi MM, et al. (2015) Maize (Zea Mays L.) Growth and Metabolic Dynamics with Plant Growth-Promoting Rhizobacteria under Salt Stresses. J Plant Pathol Microb 6: 305. doi:10.4172/2157-7471.1000305

Page 5 of 9

\begin{tabular}{|c|c|c|c|c|}
\hline \multirow{2}{*}{\multicolumn{2}{|c|}{ PGPR(Cell ml-1) }} & \multicolumn{3}{|c|}{ Saline stress $(\mathrm{mM})$} \\
\hline & & 0 & 35 & 70 \\
\hline \multicolumn{2}{|l|}{ Control } & $6.68 \pm 0.05 c$ & $3.55 \pm 0.09 i$ & $3.50 \pm 0.01 i$ \\
\hline \multirow{2}{*}{ A. vinelandii } & $10^{4}$ & $6.77 \pm 0.06 c$ & $4.31 \pm 0.05 f$ & $3.62 \pm 0.14 \mathrm{hi}$ \\
\hline & $10^{8}$ & $6.99 \pm 0.01 b$ & $4.57 \pm 0.06 e$ & $3.65 \pm 0.13 \mathrm{hi}$ \\
\hline \multirow{2}{*}{ P. fluorescens } & $10^{4}$ & $7.91 \pm 0.03 a$ & $4.81 \pm 0.03 d$ & $3.78 \pm 0.07 \mathrm{~h}$ \\
\hline & $10^{8}$ & $7.99 \pm 0.02 a$ & $4.98 \pm 0.03 d$ & $3.99 \pm 0.05 g$ \\
\hline \multirow{2}{*}{ P. putida } & $10^{4}$ & $6.68 \pm 0.06 c$ & $3.65 \pm 0.02 \mathrm{hi}$ & $3.50 \pm 0.02 i$ \\
\hline & $10^{8}$ & $6.66 \pm 0.04 c$ & $3.66 \pm 0.06 \mathrm{hi}$ & $3.62 \pm 0.07 \mathrm{hi}$ \\
\hline
\end{tabular}

Means followed by the same letter are not significantly different.

Table 8: Mean comparison and standard error of maize chlorophyll a $(\mathrm{mg} / \mathrm{g})$ under saline stress and PGPRs treatment.

\begin{tabular}{|c|c|c|c|c|}
\hline \multirow{2}{*}{\multicolumn{2}{|c|}{ PGPR(Cell ml-1) }} & \multicolumn{3}{|c|}{ Saline stress (mM) } \\
\hline & & 0 & 35 & 70 \\
\hline \multicolumn{2}{|l|}{ Control } & $3.21 \pm 0.01 b$ & $2.15 \pm 0.12 \mathrm{efg}$ & $2.10 \pm 0.13 f g$ \\
\hline \multirow{2}{*}{ A. vinelandii } & $10^{4}$ & $3.25 \pm 0.12 a b$ & $2.14 \pm 0.06 \mathrm{efg}$ & $1.91 \pm 0.06 \mathrm{~g}$ \\
\hline & $10^{8}$ & $3.32 \pm 0.04 a b$ & $2.10 \pm 0.12 \mathrm{fg}$ & $1.91 \pm 0.13 \mathrm{fg}$ \\
\hline \multirow{2}{*}{ P. fluorescens } & $10^{4}$ & $3.45 \pm 0.12 a b$ & $2.45 \pm 0.08 \mathrm{de}$ & $2.12 \pm 0.07 f g$ \\
\hline & $10^{8}$ & $3.52 \pm 0.04 a$ & $2.52 \pm 0.12 d$ & $2.01 \pm 0.12 \mathrm{fg}$ \\
\hline \multirow{2}{*}{ P. putida } & $10^{4}$ & $2.19 \pm 0.12 \mathrm{efg}$ & $2.45 \pm 0.08 \mathrm{de}$ & $2.18 \pm 0.07$ efg \\
\hline & $10^{8}$ & $2.89 \pm 0.04 c$ & $2.50 \pm 0.12 d$ & $2.23 \pm 0.12 \mathrm{def}$ \\
\hline
\end{tabular}

Means followed by the same letter are not significantly different.

Table 9: Mean comparison and standard error of maize chlorophyll b (mg/g) under saline stress and PGPRs treatment.

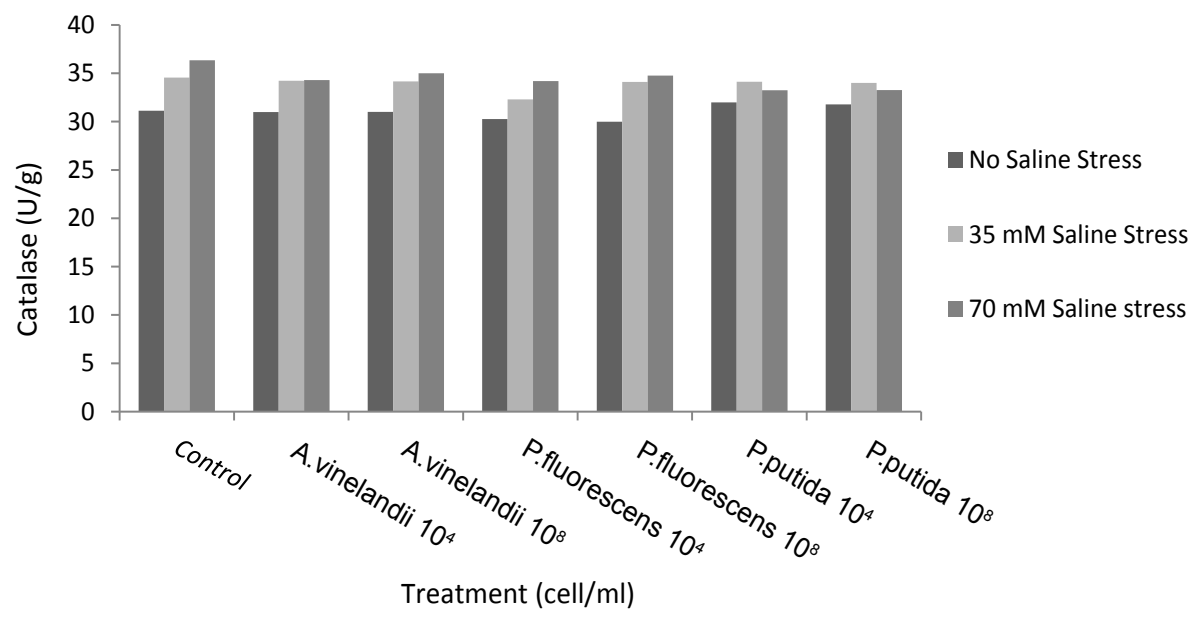

Figure 1: Effect of saline stress and PGPRs treatments on catalase production $(\mathrm{U} / \mathrm{g})$ by maize plant.

increased peroxidase concentration (Figure 2) in untreated samples. PGPRs treated samples showed irregular response to saline stress as peroxidase concentration increased at $35 \mathrm{mM}$ saline stress over the untreated samples concentrations while it was reduced at $70 \mathrm{mM}$ to minimum when compared to samples under no saline stress.

\section{Sodium potassium flux}

Root system sodium potassium balance (Table 10) was investigated in this study. It was found that Sodium potassium balance did not influence by PGPRs treatments under normal conditions. Salinity stress disturbed the $\mathrm{Na}^{+} \mathrm{K}^{+}$balance as $\mathrm{Na}^{+}$jumped up to 8.14 at $35 \mathrm{mM}$ and $9.25 \mathrm{mg} / \mathrm{g}$ at $70 \mathrm{mM}$ while $\mathrm{K}^{+}$withdrawn to be 4.43 and 4.22 at 35 and $70 \mathrm{nM}$ saline stress, respectively. A. vinelandii treatment restored $\mathrm{Na}^{+}$ $\mathrm{K}^{+}$balance at 35 and $70 \mathrm{mM}$ saline stress close to normal balance. $P$. fluorescens treatment reduced $\mathrm{Na}$ content of plant roots when compared to untreated samples at both 35 and $70 \mathrm{mM}$ saline stress; while the $\mathrm{K}^{+}$ content slightly increased. P. putida was disabled to restore the balance as $\mathrm{Na}^{+}$remained high and $\mathrm{K}^{+}$low. Sodium and potassium contents in shoot system (Table 11) were influenced by saline stress as $\mathrm{Na}^{+}$content increased up to $3.95 \mathrm{mg} / \mathrm{g}$ while the $\mathrm{K}^{+}$content reduced to $23.85 \mathrm{mg} / \mathrm{g}$. PGPRs treatments slightly reduced $\mathrm{Na}$ content of the plant shoot system and increased its $\mathrm{K}^{+}$content. Under $35 \mathrm{mM}$ saline stress also, $\mathrm{Na}^{+}$was slightly reduced by PGPRs treatments while the $\mathrm{K}^{+}$content increased especially by $A$. vinelandii and $P$. fluorescens. The same pattern observed at $70 \mathrm{mM}$ saline stress but both $\mathrm{Na}^{+}$reductio and $\mathrm{K}^{+}$improvement were limited.

\section{Discussion}

The effect of three PGPRs (Pseudomonas fluorescens, Pseudomonas putida and Azotobacter vinelandii) application on the Maize (Zea mays L.) growth and its allied attributes was assessed under saline stress in the current study. The results showed that the application of PGPRs significantly increased the shoot and root growth as compared to untreated plants. Parida and Das [37] reported that, the negative effects 
Citation: Abd El-Ghany TM, Masrahi YS, Mohamed A, Abboud A, Alawlaqi MM, et al. (2015) Maize (Zea Mays L.) Growth and Metabolic Dynamics with Plant Growth-Promoting Rhizobacteria under Salt Stresses. J Plant Pathol Microb 6: 305. doi:10.4172/2157-7471.1000305

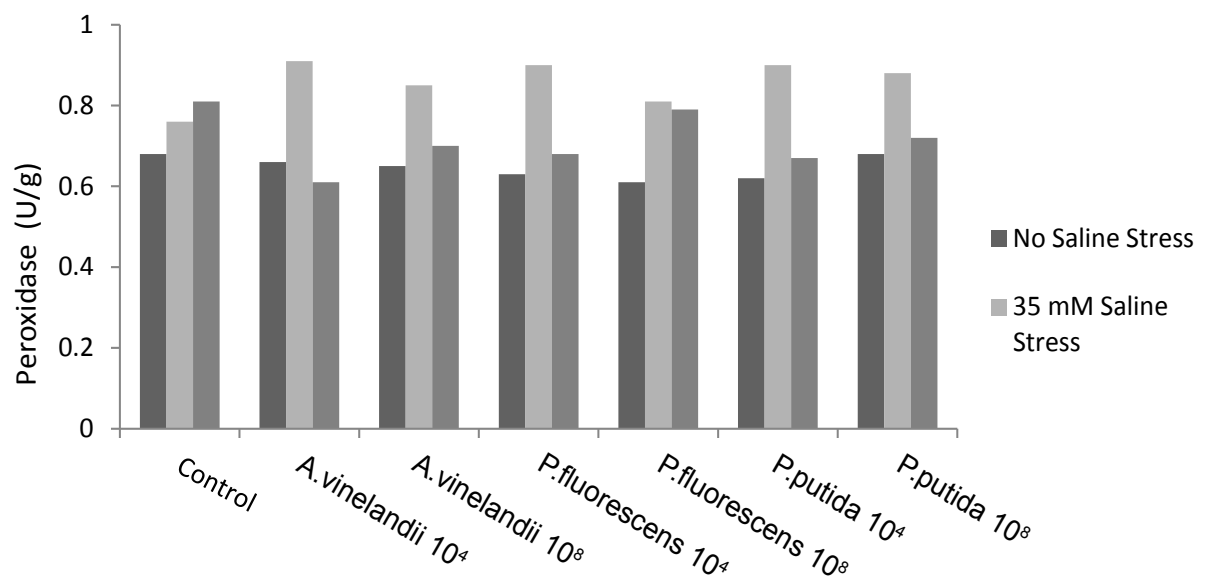

Treatment (cell/ml)

Figure 2: Effect of saline stress and PGPRs treatments on peroxidase production $(\mathrm{U} / \mathrm{g})$ by maize plant.

\begin{tabular}{|c|c|c|c|c|c|c|c|}
\hline \multirow{2}{*}{\multicolumn{2}{|c|}{ PGPR(cell ml-1) }} & \multicolumn{2}{|c|}{0} & \multicolumn{2}{|c|}{35} & \multicolumn{2}{|c|}{70} \\
\hline & & $\mathrm{Na}^{+}$ & $\mathbf{K}^{+}$ & $\mathrm{Na}^{+}$ & $\mathbf{K}^{+}$ & $\mathrm{Na}^{+}$ & $\mathbf{K}^{+}$ \\
\hline \multicolumn{2}{|l|}{ Control } & $5.78 \pm 0.032 h$ & $6.51 \pm 0.01 \mathrm{e}$ & $\begin{array}{c}8.14 \pm \\
0.027 b c\end{array}$ & $4.43 \pm 0.031$ & $9.25 \pm 0.088 a$ & $4.22 \pm 0.01 \mathrm{~m}$ \\
\hline \multirow{2}{*}{ A. vinelandii } & $10^{4}$ & $5.62 \pm 0.024 h$ & $7.07 \pm 0.04 b$ & $\begin{array}{l}6.37 \pm \\
0.032 f\end{array}$ & $6.31 \pm 0.05 f$ & $7.24 \pm 0.038 \mathrm{e}$ & $5.13 \pm 0.01 \mathrm{~g}$ \\
\hline & $10^{8}$ & $5.38 \pm 0.029 i$ & $8.07 \pm 0.10 a$ & $\begin{array}{l}6.11 \pm \\
0.009 \mathrm{~g}\end{array}$ & $6.22 \pm 0.05 f$ & $7.19 \pm 0.003 e$ & $5.20 \pm 0.03 \mathrm{~g}$ \\
\hline \multirow{2}{*}{ P. fluorescens } & $10^{4}$ & $5.63 \pm 0.022 h$ & $6.59 \pm 0.02 e$ & $\begin{array}{l}6.32 \pm \\
0.052 f\end{array}$ & $4.75 \pm 0.03 i$ & $7.21 \pm 0.006 e$ & $4.54 \pm 0.021$ \\
\hline & $10^{8}$ & $5.39 \pm 0.030 \mathrm{i}$ & $6.96 \pm 0.01 c$ & $\begin{array}{l}6.38 \pm \\
0.262 f\end{array}$ & $4.91 \pm 0.01 \mathrm{~h}$ & $7.21 \pm 0.022 \mathrm{e}$ & $4.44 \pm 0.011$ \\
\hline \multirow{2}{*}{ P. putida } & $10^{4}$ & $5.72 \pm 0.007 \mathrm{~h}$ & $6.53 \pm 0.01 e$ & $\begin{array}{l}8.04 \pm \\
0.031 c\end{array}$ & $4.60 \pm 0.02 \mathrm{jk}$ & $8.28 \pm 0.044 b c$ & $4.43 \pm 0.041$ \\
\hline & $10^{8}$ & $5.67 \pm 0.022 h$ & $6.75 \pm 0.03 d$ & $\begin{array}{l}7.77 \pm \\
0.075 d\end{array}$ & $4.66 \pm 0.02 i \mathrm{j}$ & $7.83 \pm 0.092 d$ & $4.44 \pm 0.031$ \\
\hline
\end{tabular}

$\mathrm{Na}^{+}$means followed by the same letter are not significantly different; $\mathrm{K}^{+}$means followed by the same letter are not significantly different.

Table 10: Mean comparison and standard error of maize root system $\mathrm{Na}^{+}$and $\mathrm{K}^{+}$content $(\mathrm{mg} / \mathrm{g})$ under saline stress and PGPRs treatment.

\begin{tabular}{|c|c|c|c|c|c|c|c|}
\hline \multirow{2}{*}{\multicolumn{2}{|c|}{ PGPR(cell ml-1) }} & \multicolumn{2}{|c|}{0} & \multicolumn{2}{|c|}{35} & \multicolumn{2}{|c|}{70} \\
\hline & & $\mathrm{Na}^{+}$ & $\mathbf{K}^{+}$ & $\mathrm{Na}^{+}$ & $\mathbf{K}^{+}$ & $\mathrm{Na}^{+}$ & $\mathbf{K}^{+}$ \\
\hline \multicolumn{2}{|l|}{ Control } & $2.95 \pm 0.03 \mathrm{gh}$ & $25.28 \pm 0.04 g$ & $3.51 \pm 0.03 c$ & $23.76 \pm 0.03 i$ & $3.95 \pm 0.08 a$ & $23.85 \pm 0.08 i$ \\
\hline \multirow{2}{*}{ A. vinelandii } & $10^{4}$ & $2.29 \pm 0.03 j$ & $27.54 \pm 0.27 d$ & $3.15 \pm 0.03 e f$ & $28.57 \pm 0.01 c$ & $3.83 \pm 0.03 b$ & $26.23 \pm 0.12 \mathrm{ef}$ \\
\hline & $10^{8}$ & $2.15 \pm 0.01 \mathrm{k}$ & $31.91 \pm 0.08 a$ & $3.12 \pm 0.01 f$ & $29.25 \pm 0.04 b$ & $3.32 \pm 0.01 d$ & $26.54 \pm 0.03 e$ \\
\hline \multirow{2}{*}{ P. fluorescens } & $10^{4}$ & $2.31 \pm 0.03 j$ & $27.90 \pm 0.03 d$ & $3.15 \pm 0.03 e f$ & $28.47 \pm 0.07 c$ & $3.81 \pm 0.05 b$ & $26.16 \pm 0.08 \mathrm{ef}$ \\
\hline & $10^{8}$ & $2.23 \pm 0.09 j \mathrm{k}$ & $32.17 \pm 0.41 a$ & $3.11 \pm 0.01 f$ & $29.31 \pm 0.05 b$ & $3.33 \pm 0.02 d$ & $26.42 \pm 0.07 e f$ \\
\hline \multirow{2}{*}{ P. putida } & $10^{4}$ & $2.84 \pm 0.03 h$ & $26.05 \pm 0.07 f$ & $3.24 \pm 0.04 \mathrm{de}$ & $24.42 \pm 0.05 h$ & $3.54 \pm 0.06 c$ & $23.94 \pm 0.07 i$ \\
\hline & $10^{8}$ & $2.57 \pm 0.01 i$ & $27.56 \pm 0.06 d$ & $2.98 \pm 0.05 \mathrm{gh}$ & $26.28 \pm 0.03 \mathrm{ef}$ & $3.56 \pm 0.02 c$ & $24.38 \pm 0.04 h$ \\
\hline
\end{tabular}

$\mathrm{Na}^{+}$means followed by the same letter are not significantly different; $\mathrm{K}^{+}$means followed by the same letter are not significantly different.

Table 11: Mean comparison and standard error of maize shoot system $\mathrm{Na}^{+}$and $\mathrm{K}^{+}$content $(\mathrm{mg} / \mathrm{g})$ under saline stress and PGPR treatment

of salinity stress on plant-growth include a reduction in growth rate and biomass, shorter stature, smaller leaves, osmotic effects, nutritional deficiency and mineral disorders. Therefore, according to Bacilio et al. [38] the use of PGPR to promote plant-growth in saline conditions is an important technology. The three PGPRs inocula exhibited a different pattern of shoot growth under both normal and salinity stressed conditions. Plant height, dry weight, stems diameter,and leaf surface area were clearly improved by PGPRs treatments in normal conditions. In fact, field trials have demonstrated that, inoculation with Azotobacter has beneficial effects on plant yields, due to the increase of fixed nitrogen content in agricultural soil [28,39-43], and to the microbial secretion of stimulating hormones, like gibberellins, auxins and cytokinins $[44,45]$. Our results showed that $P$. fluorescens was the best inoculum for Zea mays growth enhancing. These results confirm previous findings where the enhancing effect of Zea mays inoculation with $P$. fluorescens on dry weight and yield of maize was reported $[3,46]$, increase in plant height, root weight and total biomass were observed in response to inoculation.

Reduction in plant growth parameters under salt stress condition were recorded. Salinity is one of serious environmental problems that cause reduction in plant growth and yield productivity in irrigated 
areas of arid and semi-arid regions of the world [37]. The obtained adverse effects of salt stress on the Zea mays L. growth was alleviated by the PGPRs inoculation and decreased with concentration of inoculants. From the current study the effect of $P$. fluorescens was more pronounced than that of other two PGPRs. Our results confirm previous findings that inoculated plants grew better and had higher biomass compared to non-inoculated plants under salt stress conditions [38,47-49]. Jagnow [50] found that wheat and maize inoculated with Azotobacter increases both the plant biomass of the above ground (26-50\%) and the yield (19$30 \%)$. Recently, Zafar-ul-Hye et al. [51] found that maize productivity increased under salt stress inoculated with $P$. syringae and $P$. fluorescens.

Plants inoculated with PGPRs showed higher protein and carbohydrate content compared to control plants. Thus inoculation with $P$. fluorescens induced Zea myes L soluble protein and Carbohydrate yields (33.17 and $82.20 \mathrm{mg} / \mathrm{gm}$ respectively) compared with control (26.17 and $79.20 \mathrm{mg} / \mathrm{gm}$ respectively). On the other hand, under salt stress and without $P$. fluorescens treatment plant contents of protein and carbohydrate were decreased. The inoculation with $P$. fluorescens induce synthsis of protein and carbohydrate. Similar effects where also showed by the A. vinelandii and P. putida. Usually the increase of protein yield is related to higher nitrogen fixation activities, this knowledge was confirmed with many authors [52-54]. On the other hand, lipid content was observed to increase under salinity stress and reduced when PGPRs treatments were applied. The results indicate that $P$. fluorescens increased chlorophyll a and b (7.99 and $3.52 \mathrm{mg}$ respectively) of Zea mays L. compared with the control (6.68 and $3.21 \mathrm{mg}$ respectively) while $A$. vinelandii and $P$. putida were less effective on chlorophyll contents. Our results showed the co-inoculation of stress Zea mays L. markedly stimulated chlorophyll $\mathrm{a}$ and $\mathrm{b}$ content as compared to plants cultivated under salt stress without inoculation especially at low concentration $35 \mathrm{mM} \mathrm{Na} \mathrm{Cl}$. The effect of salinity on the synthesis of chlorophyll depended on the specific concentration of $\mathrm{NaCl}$. Nevertheless, the inoculation with PGPRs of current study enhanced the content of chlorophyll revealing a positive effect on growth and plant development. A similar trend has also been observed in other researchers $[12,55,56]$. In the present investigation, the responses of Zea maysL. plant to high level of salinity were reflected by increased of catalase and peroxidase activities. Mittler [57] stated that antioxidant enzyme activities are usually affected by salinity and used as indicators of oxidative stress in plants. To protect against oxidative stress, plant cells produce both antioxidant enzymes such as peroxidase and catalase, and non-enzymatic antioxidants such as ascorbate, glutathione and tocopherol [58]. The results showed that the exogenous application of PGPRs decreased catalase and peroxidase activities of cultivated Zea mays under salt stress. A similar trend has also been observed in other researchers $[59,60]$.

Salinity causes an imbalance in the ion flux inside plants. The present results showed that during salinity stress, the plants had higher $\mathrm{Na}^{+}$and lower $\mathrm{K}^{+}$contents, compared with control plant in root and shoot system (Tables 10 and 11). This is also according to the results [61], salinity increases the uptake of $\mathrm{Na}^{+}$or decreases the uptake $\mathrm{K}^{+}$ which lead to nutritional imbalances. Accumulation of excess $\mathrm{Na}^{+}$may cause metabolic disturbance in processes where low $\mathrm{Na}^{+}$and high $\mathrm{K}^{+}$are required for optimum plant function [62]. Increased $\mathrm{K}^{+}$concentration under salinity conditions may help to decrease $\mathrm{Na}^{+}$uptake and this can indirectly maintain the growth of the plant [63]. Based on the results obtained, applying PGPRs treatment significantly increased the $\mathrm{K}^{+}$ content of maize under salt stress conditions. The higher $\mathrm{K}^{+}$uptake may demonstrate the role of $\mathrm{K}^{+}$in salt tolerance. This is also according to the results of [64] where PGPR strains from Azotobacter sp. increased the maize plant growth and potassium and phosphorus intake under different levels of salinity stress. Recently, Sang-Mo et al. [65] found that the PGPR-applied plants had reduced sodium ion concentration; while the potassium was abundantly present as compared to control under stress of Cucumis sativus cultivation. $\mathrm{K}^{+}$play a key role in plant water stress tolerance and has been found to be the cationic solute responsible for stomata movements in response to changes in bulk leaf water status [66]. There are several reports of lower $\mathrm{Na}^{+}$concentrations in plants inoculated with PGPR under salinity conditions $[63,67,68]$.

\section{Conclusion}

P. fluorescens, A. vinelandii and P. putida had significant impact on maize growth, suggesting that can be applied as biofertilizers for improved maize production under salinity stress. Further greenhouse studies should provide more definitive information about the movement and uptake of macro-elements $\left(\mathrm{Na}^{+}\right.$and $\left.\mathrm{K}^{+}\right)$to plants with the impacts of PGPR-based inoculants.

\section{Acknowledgement}

Part of this study was conducted as part of the Project No. 30-G5-1434: "Modern integrated management of halothermophilic biofertilizers for enhancing agricultural soil fertility in Jazan", which is supported and funded by the Deanship of Scientific Research, Jazan University, KSA.

\section{References}

1. Kannaiyan S, Kumar K, Govindarajan K (2004) Biofertilizers technology for rice based cropping. Jodhpur, p: 450

2. Nuttall N (2006) Soil biodiversity key to environmentally friendly agriculture. Below ground biodiversity-from worms to bacteria-key to environmentally friendly agriculture. The 8th Conference of the Parties to the Convention on Biological Diversity, Curitiba, Brazil.

3. Shaharoona B, Arshad M, Zahir ZA, Khalid A (2006) Performance of Pseudomonas spp. containing ACC-ase for improving growth and yield of maize (Zea mays L.) in the presence of nitrogenous fertilizer. Soil Biology and Biochemistry 38: 2971-2975.

4. Awad EMM, Abd El-Hameed AM, Shall ZS (2007) Effect of glycine, lysine and nitrogen fertilizer rates on growth, yield and chemical composition of potato. $J$ Agric Sci Mansoura Univ 32: 8541-8551.

5. Faten SA, Shaheen AM, Ahmed AA, Mahmoud AR (2010) Effect of foliar application of amino acids as antioxidants on growth, yield and characteristics of Squash. Research Journal of Agriculture and Biological Science 6: 583-588.

6. Maryam S, Mohammad RH, Mohammad TD (2014) Effects of amino acids and nitrogen fixing bacteria on quantitative yield and essential oil content of basil (Ocimum basilicum). Agriculture Science Developments 3: 265-268.

7. Benizri E, Baudoin E, Guckert A (2001) Root colonization by inoculated plant growth-promoting rhizobacteria. Biocontrol Sci Techn 11: 557-574.

8. Anonymous (2000) Agricultural Statistics of Pakistan, Government of Pakistan Ministry of Food, Agriculture and Livestock, Economic Wing, Islamabad. p: 104

9. Subramaniyan V, Krishna S, Malliga P (2012) Analysis of biochemical and yield parameters of Zea Mays (Corn) cultivated in the field supplemented with coir pith based cyanobacterial biofertilizers.

10. Yao T, Yasmin S, Hafeez FY (2008) Potential role of rhizobacteria isolated from Northwestern China for enhancing wheat and oat yield.The Journal of Agricultural Science 146: 49-56.

11. Rong LY, yao T, zhao GQ, (2011) Filtration of siderophores production PGPR bacteria and antagonism against to pathogens. Plant Protection 1: 59-64.

12. Nadeem SM, Zahir ZA, Naveed M, Arshad M, Shahzad SM (2006) Variation in growth and ion uptake of maize due to inoculation with plant growth promoting rhizobacteria under salt stress. Soil \& Environ 25: 78-84.

13. Hasnain S, Sabri AN (1996) Growth stimulation of Triticum aestivum seedlings under $\mathrm{Cr}$-stress by nonrhizospheric Pseudomonas strains. In: 7th International Symposium on nitrogen fixation with non-legumes. Faisalabad, Pakistan, p: 36

14. Ashraf M, Berge SH, Mahmood OT (2004) Inoculating wheat seedling with 
Citation: Abd El-Ghany TM, Masrahi YS, Mohamed A, Abboud A, Alawlaqi MM, et al. (2015) Maize (Zea Mays L.) Growth and Metabolic Dynamics with Plant Growth-Promoting Rhizobacteria under Salt Stresses. J Plant Pathol Microb 6: 305. doi:10.4172/2157-7471.1000305

exopolysaccharide-producing bacteria restricts sodium uptake and stimulates plant growth under salt stress. Biology and Fertility of Soils 40: 157-162.

15. Sylvia DM, Fuhrmann JJ, Hartel PG (1998) Zuberer Principles and applications of soil microbiology. Prentice-Hall, Inc. Upper Saddle River, NJ, 1998:550.

16. Johri BN, Sharma A, Virdi JS (2003) Rhizobacterial diversity in India and its influence on soil and plant health. Adv Biochem Eng Biotechnol 84: 49-89.

17. Roesch LFW, Camargo FAO, Bento FM, Triplett, EW (2008) Biodiversity of diazotrophic bacteria within soil, root and stem of field grown maize. Plant Soil 302: 91-104.

18. Glick BR, Karaturovi DM, Newell PC (1995) A novel procedure for rapid isolation of plant growth-promoting pseudomonads. Can J Microbiol 41: 533-536.

19. Weller DM (1988) Biological control of soil borne plant pathogens in the rhizosphere with bacteria. Ann Rev Phytopathol 26: 379-407.

20. Kloepper JW (1997) Current status and future trends in Biocontrol Research and Development in the US. -In: International symposium on clean agriculture, Sapporo, DECD, pp: 49-52.

21. Skinner PS, Banfield JB (2005) The Role of Microorganisms in Nitrogen Fixation. Journal of Symbiotic Association 14: 600-700.

22. Yooshinan SU (2001) Biological Nitrogen Fixation. Journal of Biological Association 8: 50-55

23. Ahmed ZI, Ansar M, Tariq M, Anjum MS (2008) Effect of different Rhizobium inoculation methods on performance of lentil in pothowar region. Int $\mathrm{J}$ Agric Biol 10: 85-88.

24. Vessey JK (2003) Plant growth promoting rhizobacteria as biofertilizers. Plan Soil 255: 571-586.

25. Das K, Dang R, Shivananda TN, Sekeroglu N (2007) Influence biofertilizers on the biomass yield and nutrient content in Stevia rebaudiana Bert. grown in Indian subtropics. J Med Plants Res 1: 5-8.

26. Verma NK (2011) Integrated nutrient management in winter maize (Zea mays L.) sown at different dates. Afr J Plant Breed Crop Sci 3: 161-167.

27. Mishra OR, Tomar US, Sharama RA, Rajput AM (1995) Response of maize to chemicals and biofertilizers. Crop Research 9: 233-237.

28. Palleroni NJ (1984) Gram negative aerobic rods and cocci. In: Krieg NR, (Ed), Bergey's Manual of Systematic Bacteriology, Williams and Wilkins, Baltimore, pp: 140-199.

29. Pandey A, Sharma E, Palni L (1998) Influence of bacterial inoculation on maize in upland farming systems of the Sikkim Himalaya. Soil Biology and Biochemistry 3: 379-384.

30. Radwan FI (1998) Response of some maize cultivars to VA-mycorrhiza inoculation, biofertilization and soil nitrogen application. Alexandria $J$ Agricultural Research 43: 43-56.

31. Fares CN (1997) Growth and yield of wheat plant as affected by biofertilisation with associative, symbiontic N2-fixers and endomycorrhizae in the presence of the different P-fertilizers. Annals of Agriculture Science 42: 51-60.

32. Tan GH, Nordin MS, Kert TL, Napsiah AB, Jeffrey LSH (2009) Isolation of beneficial microbes from biofertilizer products. J Trop Agric and Fd Sc 37 103-109.

33. Hensyl WR (1994) Bergey's 'Manual of Systematic Bacteriology (9thEdn). John G Holt and Stanley, T. Williams (Eds.) Williams and Wilkins, Baltimore, Philadeiphia, Hong kong, London, Munich, Sydney, Tokyo.

34. Ryan J, Estefan G, Rashid A (2001) Soil and analysis laboratory manual. ICARDA, p: 172

35. Kar M, Mishra D (1976) Catalase, Peroxidase, and Polyphenoloxidase Activities during Rice Leaf Senescence. Plant Physiol 57: 315-319.

36. Bradford MM (1976) A rapid and sensitive method for the quantitation of microgram quantities of protein utilizing the principle of protein-dye binding. Anal Biochem 72: 248-254.

37. Hedge JE, Hofreiter BT (1962) In: Carbohydrate Chemistry, 17 (Eds. Whistler R.L. and Be Miller, J.N.), Academic Press, New York.

38. Parida AK, Das AB (2005) Salt tolerance and salinity effects on plants: a review. Ecotoxicol Environ Saf 60: 324-349.
39. Bacilio M, Rodriguez H, Moreno M, Hernandez JP, Bashan Y (2004) Mitigation of salt stress in wheat seedlings by a gfp-tagged Azospirillum lipoferum. Boil Fertil Soil 40: 188-193.

40. Sarig S, Blum A, Okon Y (1988) Improvement of the water status andyield of field-grown grain sorghum (Sorghum bicolor) by inoculation with Azospirillum brasilense. J Agric Sci 110: 271-277.

41. Zahir ZA, Arshad M, Hussain A, Sarfraz M (1996) Improving wheat yield by inoculation with Azotobacter under optimum fertilizer application. Pakistan Journal of Agricultural Science 11: 129-131.

42. Naz I, Asghari B, Bushra R, Simab P, Mazhar I, et al. (2012) Potential of Azotobacter vinelandii Khsr1 as bioinoculant. African J Biotechnol 1: 1036810372.

43. Abd El Ghany TM, Alawlaqi MM, Al Abboud MA (2013) Role of biofertilizers in agriculture: a brief review. Mycopath 11: 95-101.

44. Nalawde A, Bhalerao SA (2015) Effect of Biofertilizer (Azotobacter sp.) on the Growth of Blackgram Vigna mungo (L.) Hepper Int J Curr Res Biosci Plant Bio 2: 20-23.

45. Salmeron V, Martinez Toledo MV, Gonzalez Lopez Jb (1990) Nitrogen fixation and production of auxins, gibberellins and cytokinin by Azotobacter chroococcum strain isolated from root of Zea mays in presence of insoluble phosphate. Chemosphere 20: 417-422.

46. Gonzales-Lopez J, Martinez Toledo MV, Reina S, Salmeron V (1991) Root exudates of maize on production of auxins, gibberellins, cytokinins, amino acids and vitamins by zotobacter chroococcum chemically defined media and dialysed soil media. Toxicology and Environmental Chemistry 33: 69-78.

47. Gupta S, Arora DK, Srivastava AK (1995) Growth promotion of tomato plants by rhizobacteria and imposition of energy stress on Rhizoctonia solani. Soil Bio Biochem 27: 1051-1058.

48. Diouf D, Duponnois R, Tidiane BA, Neyra M, Lesueur D (2005) Symbiosis of Acacia auriculiformis and Acacia mangium with Arbuscular mycorrhizal fung and Bradyrhizobium spp. improves salt tolerance in greenhouse conditions. Funct Plant Biol 32: 1143-1152.

49. Ravikumar SK, Alikhan KL, Prakash S, Williams G, Gracelin NAA (2007) Growth of Avicennia marina and Ceriops decandra seedlings inoculated with halophilic azotobacters. Journal of Environmental Biology 28: 601-603.

50. Omid Y, Abolfaz B, Amin N (2013) The effects of Pseudomonas fluorescence and Rhizobium meliloti co-inoculation on nodulation and mineral nutrient contents in alfalfa (Medicago sativa) under salinity stress. Intl J Agri Crop Sci 5: 1500-1507.

51. Jagnow G (1987) Inoculation of cereal crops and forage grasses with nitrogenfixing rhizosphere bacteria: possible causes of success and failure with regard to yield response. A review - Z. Pflanzenernahr bodenkd 150: 361-368.

52. Zafar-ul-Hye M, Farooq HM, Zahir ZA, Hussain M, Hussain A (2014) application of ACC-deaminase containing rhizobacteria with fertilizer improves maize production under drought and salinity stress. Int J Agric Biol 16: 591-596.

53. Zhang F, Dashti N, Hynes RK, Smith DI (1996) Plant Growth Promoting Rhizobacteria and Soybean [ Glycine max (L.) Merr.] Nodulation and Nitrogen Fixation at Suboptimal Root Zone Temperatures. Ann Bot 77: 453-460.

54. Marius S, Neculai M, Vasile S, Marius M (2013) Effects of inoculation with plant growth promoting rhizobacteria on photosynthesis, antioxidant status and yield of runner bean. Romanian Biotechnological Letters 18: 8132-8143.

55. Prathibha KS, Siddalingeshwara KG (2013) Effect of plant growth promoting Bacillus subtilis and Pseudomonas fluorescence as Rhizobacteria on seed quality of sorghum. Int J Curr Microbiol App Sci 2: 11-18

56. Swedrzynska D, Sawicka A (2000) Effect of Inoculation with Azospirillum brasilense on Development and Yielding of Maize (Zea mays ssp. Saccharata L.) under Different Cultivation Conditions. Polish Journal of Environmental Studies 9: 505-509.

57. Parida A, Das AB, Mittra B (2004) Effects of salt on growth, ion accumulation, photosynthesis and leaf anatomy of the mangrove, Bruguiera parviflora. Trees Struct Funct 18: 167-174

58. Mittler R (2002) Oxidative stress, antioxidants and stress tolerance. Trends Plant Sci 7: 405-410.

59. del Río LA, Corpas FJ, Sandalio LM, Palma JM, Barroso JB (2003) Plant 
Citation: Abd El-Ghany TM, Masrahi YS, Mohamed A, Abboud A, Alawlaqi MM, et al. (2015) Maize (Zea Mays L.) Growth and Metabolic Dynamics with Plant Growth-Promoting Rhizobacteria under Salt Stresses. J Plant Pathol Microb 6: 305. doi:10.4172/2157-7471.1000305

Page 9 of 9

peroxisomes, reactive oxygen metabolism and nitric oxide. IUBMB Life 55: 71 . 81.

60. Shaik ZA, Vardharajula S, Minakshi G, Venkateswar RL, Bandi V (2011) Effect of inoculation with a thermotolerant plant growth promoting Pseudomonas putida strain AKMP7 on growth of wheat (Triticum spp.) under heat stress, Journal of Plant Interactions 6: 239-246.

61. Yachana J, Subramanian RB (2013) Paddy plants inoculated with PGPR show better growth physiology and nutrient content under saline conditions. Chilean journal of agricultural research 73: 213-219.

62. Yildirim E, Taylor AG, Spittler TD (2006) Ameliorative effects of biological treatments on growth of squash plants under salt stress. Sci Hortic 111: 1-6.

63. Glenn EP, Brown JJ, Blumwald E (1999) Salt tolerance and crop potential of halophytes. Crit Rev Plant Sci 18: 227-255.

64. Giri B, Mukerji KG (2004) Mycorrhizal inoculant alleviates salt stress in Sesbania aegyptiaca and Sesbania grandiflora under field conditions, evidence for reduced sodium and improved magnesium uptake. Mycorrhiza 14: 307-312.

65. Rojas-Tapias D, Moreno-Galván A, Pardo-Díaz S, Obando M, Rivera D, et al (2012) Effect of inoculation with plant growth-promoting bacteria (PGPB) on amelioration of saline stress in maize (Zea mays). Appl Soil Ecol 61: 264-272.

66. Sang-Mo K, Abdul Latif K, Muhammad W, Young-Hyun Y, Jin-Ho K, et al. (2014) Plant growth-promoting rhizobacteria reduce adverse effects of salinity and osmotic stress by regulating phytohormones and antioxidants in Cucumis sativus. Journal of Plant Interactions 9: 673-682.

67. Caravaca F, Figueroa D, Barea JM, Azcón-Aguilar C, Roldán A (2004) Effect of mycorrhizal inoculation on the nutrient content, gas exchange and nitrate reductase activity of Retama sphaerocarpa and Olea europaea subsp. sylvestris under drought stress. Journal of Plant Nutrients 27: 57-74.

68. Sharifi M, Ghorbanli M, Ebrahimzadeh H (2007) Improved growth of salinitystressed soybean after inoculation with salt pre-treated mycorrhizal fungi. J Plant Physiol 164: 1144-1151. 